\title{
Copper(I)-Catalyzed Enantioselective Boryl Substitution of Allyl Acylals: An Efficient Approach for Enantioenriched $\alpha$-Chiral Y-Acetoxyallylboronates
}

\author{
Yuta Takenouchi \\ Ryoto Kojima \\ Riko Momma \\ Hajime Ito*
}

Division of Applied Chemistry and Frontier Chemistry Center, Faculty of Engineering, Hokkaido University, Sapporo, Hokkaido, 060-8628, Japan

hajito@eng.hokudai.ac.jp

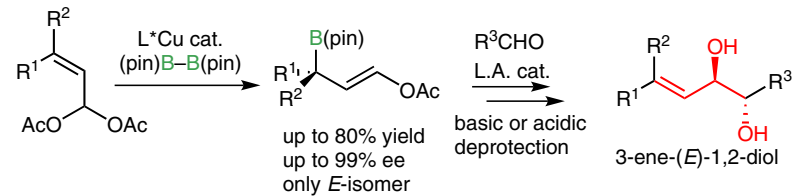

activity toward the boryl copper nucleophile. In addition, this reaction required harsh reaction conditions to allow for the removal of the benzyl groups from the monoprotected 1,2-diols, which were obtained by the allylation of aldehydes with the corresponding $\gamma$-alkoxyallylboronates. Furthermore, the route required for the synthesis of the dibenzyl acetal substrates showed limited substrate scope, as well as being a laborious and time-consuming procedure. ${ }^{6}$

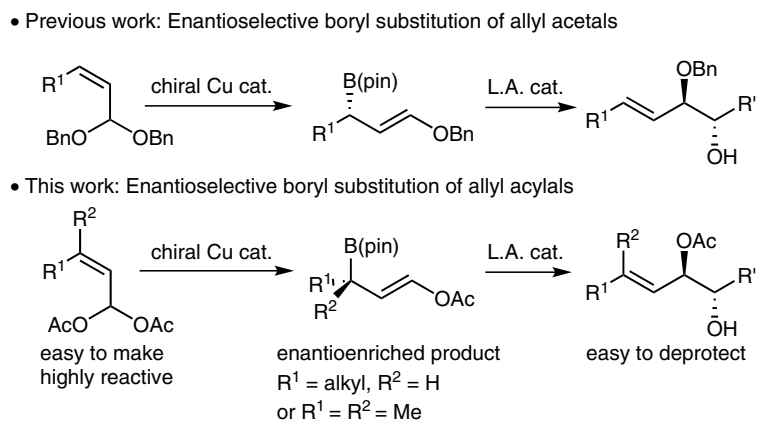

Scheme 1 Copper(I)-catalyzed enantioselective boryl substitution of allyl acylals

To address these issues, we focused on allyl acylals as alternative substrates for the copper-catalyzed boryl substitution reaction. Allyl acylals have been shown to be well suited to nucleophilic substitution reactions, such as palladium-catalyzed asymmetric alkylations ${ }^{7}$ or Lewis acid catalyzed cyanation. ${ }^{8}$ We therefore expected that allyl acylals would be more reactive than allyl acetals toward nucleophilic boryl substitution reactions because the acetoxy group in the former is more electron withdrawing than the ether group in the latter, making the LUMO of the allyl acylal substrate lower in energy and more reactive toward a nucleophilic boryl copper intermediate. 
Furthermore, acetyl groups can be removed under milder conditions than those required to remove ether groups, making this process more efficient than our previous method. ${ }^{9}$ Notably, a facile synthetic method has been reported for the direct construction of allyl acylals from aldehydes and acetic anhydride using an acid catalyst. ${ }^{10}$

Herein, we report the enantioselective synthesis of $\alpha$ chiral $\gamma$-acetoxyallylboronates using a chiral copper catalyst and bis(pinacolato)diboron $\left[\mathrm{B}_{2}(\text { pin })_{2}\right]$ as a boron source. Notably, this reaction was successfully applied to a wide range of allyl acylal substrates, including sterically hindered compounds, to give the desired products in good yields.

Initial optimization studies focused on the $E / Z$ selectivity and enantioselectivity of the copper(I)-catalyzed boryl substitution of an allyl acylal to give the corresponding allylboronate. The reaction of acylal $(Z)-\mathbf{1 a}$ with $\mathrm{B}_{2}(\mathrm{pin})_{2}$ in the presence of $\mathrm{CuCl} /(R, R)$-BenzP* as a ligand (5 mol\%) and $\mathrm{KOt}$-Bu as a base (1 equiv) in THF or toluene afforded mixtures of the corresponding $E$ and $Z$ products (Table 1, entries 1 and $2) .{ }^{11}$ In our previous study involving the borylation of allyl acetals, we only ever observed the formation of the $E$ iso- mer as a single product, which we attributed to the substrate undergoing an anti $\mathrm{S}_{\mathrm{N}} 2$ ' reaction mechanism with a fixed conformation because of the 1,3-allylic strain of the substrate (see the Supporting Information), ${ }^{5,12}$

The use of 1,3-dimethyl-2-imidazolidinone (DMI) as a solvent provided the $E$ product with high $E / Z$ selectivity and excellent enantioselectivity (73\% yield, $E / Z=98: 2$, 89\% ee; Table 1, entry 3). Several other chiral ligands, including $(R, R)$-QuinoxP*, $(R)$-Segphos, and $(R, R)$-Me-Duphos, were also tested, but resulted in poor yields and $E / Z$ selectivities (Table 1 , entries 4-6). The amounts of base and $B_{2}(\text { pin })_{2}$ added to the reaction also had a considerable impact in the reactivity. For example, the use of a catalytic amount of $\mathrm{KOt}$-Bu (10 mol\%) yielded a trace amount of the desired product, whereas the use of small excesses of $\mathrm{KOt}-\mathrm{Bu}$ (1.5 equiv) and $\mathrm{B}_{2}$ (pin $)_{2}$ (2.0 equiv) resulted in high yield with excellent $E / Z$ selectivity and enantioselectivity (79\% yield, $E / Z=>99: 1,95 \%$ ee; Table 1 , entry 8$).{ }^{13}$

As shown in Scheme 2, various $\alpha$-chiral $\gamma$-acetoxyallylboronates were obtained in high yields and enantioselectivities under the optimized reaction conditions. Furthermore,

Table 1 Optimization of the Reaction Conditions for the Copper(I)-Catalyzed Enantioselective Boryl Substitution of Allyl Acylal (Z)-1 $\mathrm{a}^{\mathrm{a}}$
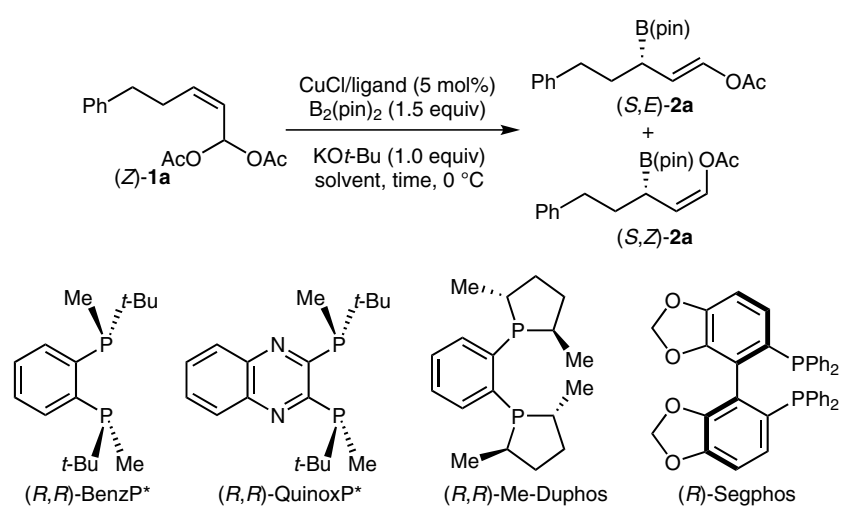

\begin{tabular}{|c|c|c|c|c|c|c|}
\hline Entry & Solvent & Ligand & Time (h) & $E \mid Z^{b}$ & Yield $(\%)^{c}$ & ee $(\%)^{d}$ \\
\hline 1 & THF & $(R, R)$-Benz ${ }^{*}$ & 30 & $82: 18$ & 78 & 93 \\
\hline 2 & toluene & $(R, R)$-BenzP* & 48 & $76: 24$ & 74 & 92 \\
\hline 3 & DMI & $(R, R)$-Benz ${ }^{*}$ & 45 & $98: 2$ & 73 & 89 \\
\hline $4^{e}$ & DMI & $(R, R)$-QuinoxP* & 24 & $90: 10$ & 30 & - \\
\hline $5^{e}$ & DMI & (R)-Segphos & 24 & $87: 13$ & 23 & - \\
\hline $6^{e}$ & DMI & $(R, R)$-Me-Duphos* & 24 & $79: 21$ & 30 & - \\
\hline $7^{f}$ & DMI & $(R, R)$-BenzP* & 24 & - & trace & - \\
\hline $8^{g}$ & DMI & $(R, R)$-Benz $\mathrm{P}^{*}$ & 28 & $>99: 1$ & 79 & 95 \\
\hline
\end{tabular}

${ }^{a}$ Reagents and conditions: $\mathrm{CuCl}(0.01 \mathrm{mmol})$, ligand (0.01 mmol), (Z)-1a (0.2 mmol), $\mathrm{B}_{2}(\text { pin })_{2}(0.3 \mathrm{mmol})$, and $\mathrm{KOt}$ - Bu $(0.2 \mathrm{mmol})$ in solvent $(0.4 \mathrm{~mL})$ at $0{ }^{\circ} \mathrm{C}$.

b $T$ he $E / Z$ selectivity was determined by GC.

c NMR yield.

d The ee values of the products were determined by HPLC analysis.

e The ee value of the major product was difficult to determine using HPLC analysis because both $\mathrm{SiO}_{2}$ and chiral column chromatography resulted in an insuffi-

cient separation of the major product and the unconsumed substrate.

f $10 \mathrm{~mol} \%$ of $\mathrm{KO} t$-Bu was used.

g 2.0 equiv of $B_{2}(\text { pin })_{2}$ and 1.5 equiv of KOt-Bu were used; 0.5 mmol scale. 
several optically active products bearing an alkyl substituent (e.g., $\mathrm{R}=\mathrm{Me}$, hexyl, methylcyclopentyl) were obtained in high yields and enantioselectivities [ $(S, E)-\mathbf{2 b}, 80 \%$ yield, 99\% ee; (S,E)-2c, $80 \%$ yield, $98 \%$ ee; (S,E)-2d, $76 \%$ yield, $94 \%$ ee]. This reaction also showed good functional-group tolerance, as exemplified by the boryl substitution of substrates bearing a silyl ether or acetoxy group, which proceeded in high yield and excellent enantioselectivity without any degradation of the functional groups $[(S, E)-\mathbf{2 e}, 77 \%$ yield, 93\% ee; $(S, E)-2 f$, $60 \%$ yield, $93 \%$ ee; $(S, E)-\mathbf{2 g}$, $62 \%$ yield, $95 \%$ ee]. $\sigma$-Branched allyl acylals $[(Z)-\mathbf{1 h}$ and $(Z)-\mathbf{1 i}]$, which have steric congestion around their $\mathrm{C}=\mathrm{C}$ bond, also reacted smoothly to afford the corresponding borylated products ( $58 \%$ and $42 \%$ yield, respectively), but the enantiopurities of these products were unfortunately low (59\% and 55\% ee, respectively), compared with $\mathbf{2 b}$ and $\mathbf{2 c}$. The borylation of the $E$ substrate $(E)-\mathbf{1} \mathbf{j}(E / Z=95: 5)$ proceeded with poor enantioselectivity to give the corresponding product with the opposite absolute configuration for the boron atom $[(R, E)-\mathbf{2} \mathbf{j}$, $81 \%$ yield, $74 \%$ ee, $E / Z=91: 9]$.

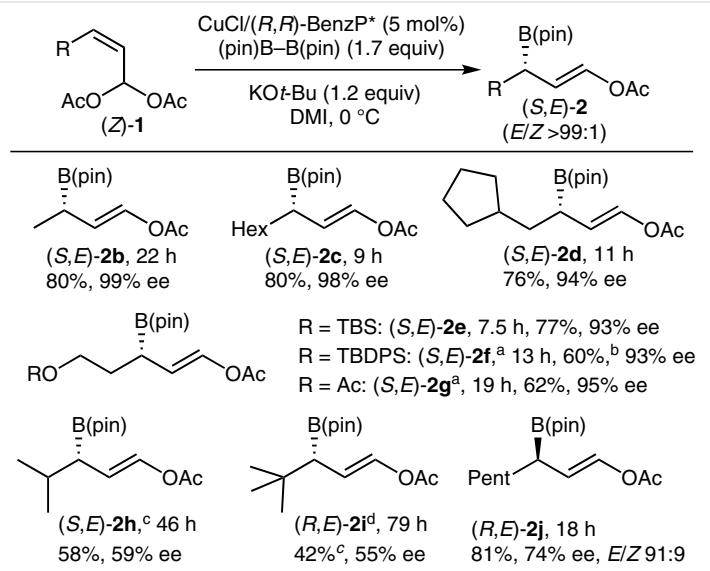

Scheme 2 Substrate scope of the copper(I)-catalyzed enantioselective boryl substitution of allyl acylal (Z)-1. Reagents and conditions: $\mathrm{CuCl}$ (0.025 mmol), (R,R)-BenzP* (0.025 mmol), (Z)-1 (0.5 mmol), $\mathrm{B}_{2}(\text { pin })_{2}$ $(0.85 \mathrm{mmol})$ and $\mathrm{KOt}-\mathrm{Bu}(0.6 \mathrm{mmol})$ in DMI $(1.0 \mathrm{~mL})$ at $0{ }^{\circ} \mathrm{C}$. The ee values of the products were determined by HPLC analysis. ${ }^{a} 1.5$ equiv of $\mathrm{KOt}$-Bu and 2.0 equiv of $\mathrm{B}_{2}(\text { pin })_{2}$ were used. ${ }^{\mathrm{b}} \mathrm{NMR}$ yield. ${ }^{\mathrm{c}} \mathrm{THF}(0.3 \mathrm{~mL})$ and DMI $(0.3 \mathrm{~mL})$ were used as a solvent; $10 \mathrm{~mol} \%$ of $\mathrm{CuCl}$ and $(R, R)$ BenzP* were used. ${ }^{d}$ THF $(1.0 \mathrm{~mL})$ was used as a solvent; $15 \mathrm{~mol} \%$ of $\mathrm{CuCl}$ and $(R, R)$-Benz $\mathrm{P}^{*}$ were used; $0.2 \mathrm{mmol}$ scale.

We then proceeded to compare the reactivities of the allyl acetal and acylal substrates. Ally acetal $\mathbf{3}$ and acylal $\mathbf{1 k}$, which both have a trisubstituted alkene moiety, were selected as model substrates. The boryl substitution of acetal 3 provided only a trace amount of the corresponding borylated product $(E)-\mathbf{4}$ in 4 hours. Even after an extended reaction time ( $>24 \mathrm{~h}$ ), the allyl acetal 3 remained largely intact. The low conversion of the acetal substrate was attributed to steric hindrance around the $\mathrm{C}=\mathrm{C}$ double bond of the sub- strate and the poor leaving group ability of the methyl ether group compared with the acetyl group. In contrast, the acylal substrate $1 \mathbf{k}$ reacted much more effectively than the acetal to give the borylated product in $49 \%$ yield after 24 hours (Scheme 3). These results therefore demonstrate that acylal substrates can undergo allyl substitution much more effectively than the corresponding acetals.

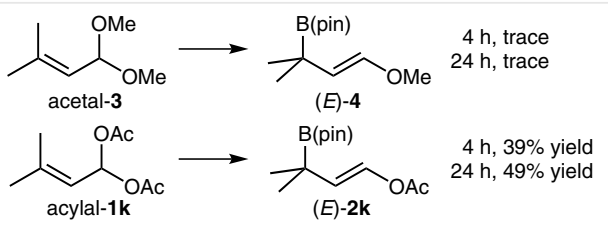

Scheme 3 y-Borylation of trisubstituted allyl acetal and acylal with Cu$\mathrm{Cl} /$ Xantphos catalyst system. Reagents and conditions: $\mathrm{CuCl} / \mathrm{Xantphos}(5$ mol\%), $\mathrm{B}_{2}$ (pin) $)_{2}$ (1.5 equiv), KOt-Bu (1.0 equiv), THF, $30^{\circ} \mathrm{C}$.

The allylboronates $(S, E)$-2f prepared using our new method were subsequently applied to the stereoselective allylation of aldehyde (Scheme 4). Octynal was successfully allylated with boronate $(S, E)-\mathbf{2 f}$ in the presence of $\mathrm{ZnBr}_{2}$, which was added as a Lewis acid catalyst. ${ }^{14,15}$ We previously found that $\mathrm{ZnBr}_{2}$ is an efficient catalyst for enhancing the stereoselectivity and accelerating the reaction rate for the allylation of aldehydes with $\gamma$-alkoxyallylboronates. ${ }^{5}$ With this in mind, we investigated the reaction of octynal with $(S, E)-2 \mathbf{f}$ in the presence of $\mathrm{ZnBr}_{2}$. Pleasingly, this reaction provided the desired product in high stereoselectivity and good $E / Z$ selectivity $[(E)$-anti-5, $68 \%$ yield, $96 \%$ ee, $E / Z=$ 94:6].

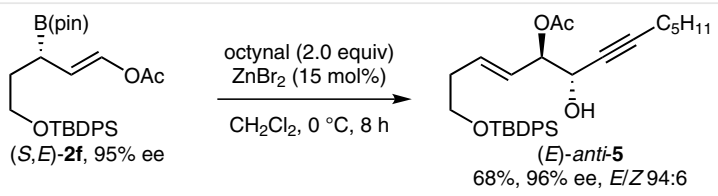

Scheme 4 Aldehyde allylation with optically active $\gamma$-acetoxyallylboronate (S,E)-2f. Reagents and conditions: $(S, E)$-2f $(0.2 \mathrm{mmol})$, aldehyde $(0.4$ $\mathrm{mmol})$, and dry $\mathrm{ZnBr}_{2}(15 \mathrm{~mol} \%)$ in $\mathrm{CH}_{2} \mathrm{Cl}_{2}(0.4 \mathrm{~mL})$ at $0{ }^{\circ} \mathrm{C}$. Dry $\mathrm{ZnBr}_{2}$ was required to obtain high levels of stereoselectivity. (S,E)-2f with $95 \%$ ee was used. The minor syn isomers of $\mathbf{5}$ were present in trace amounts, which were detected by ${ }^{1} \mathrm{H}$ NMR analysis of the crude reaction mixtures. The ee value of the major product was determined by HPLC analysis. The $E / Z$ ratios of the anti product were determined by ${ }^{1} \mathrm{H}$ NMR and HPLC analyses.

The acetyl group in the allylation product (E)-anti-5 was readily removed under acidic conditions (Scheme 5, conditions A) to give the corresponding diol in 73\% yield without lowering its enantiomeric purity. The acetyl group was also removed under basic conditions to afford the desired product (E)-anti-6 in good yield without any degradation of the functional group or loss of optical purity (conditions B). 


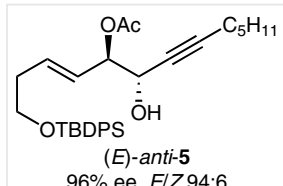

$96 \%$ ee, E/Z94:6

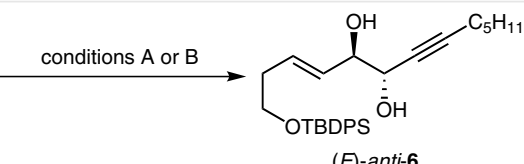

$(E)$-anti-6

A) $73 \%$ yield, $96 \%$ ee, E/Z $89: 11$ B) $61 \%$ yield, $96 \%$ ee, $E / Z 89: 11$

Scheme 5 Deprotection of the acetyl group in the allylation products under acidic and basic conditions. Conditions $\mathrm{A}$ : $\mathrm{Sc}(\mathrm{OTf})_{3}$ (2.0 equiv), $\mathrm{MeOH}-\mathrm{H}_{2} \mathrm{O}$, r.t., 24 h; conditions $\mathrm{B}: \mathrm{K}_{2} \mathrm{CO}_{3}$ (2.0 equiv), $\mathrm{MeOH}-\mathrm{H}_{2} \mathrm{O}$, r.t., $30 \mathrm{~min}$.

In summary, we have developed a new method for the asymmetric synthesis of chiral $\gamma$-acetoxyallylboronates via the copper(I)-catalyzed boryl substitution of allyl acylals. The resulting allylboronates were used to achieve the highly stereoselective allylation of aldehydes. Furthermore, the acetyl groups of the allylated products were readily removed under basic and acidic conditions to give the corresponding 1,2-diols. This reaction therefore represents a useful method for the synthesis of 3-(E)-alkenyl-anti-1,2diols.

\section{Acknowledgment}

This study was financially supported by the MEXT (Japan) program (Strategic Molecular and Materials Chemistry through Innovative Coupling Reactions) of Hokkaido University, as well as the JSPS (KAKENHI Grant Numbers 15H03804 and 15K13633).

\section{Supporting Information}

Supporting information for this article is available online at http://dx.doi.org/10.1055/s-0036-1588354.

\section{References and Notes}

(1) (a) Hall, D. G. Boronic Acid: Preparation, Applications in Organic Synthesis and Medicine; Wiley-VCH: Weinheim, 2005. (b) Hoffmann, R. W. Angew. Chem., Int. Ed. Engl. 1982, 21, 555. (c) Hall, D. G. Synlett 2007, 11, 1644.

(2) (a) Hoffmann, R. W.; Zeiss, H. J. Angew. Chem., Int. Ed. Engl. 1979, 18, 306. (b) Brown, H. C.; Bhat, K. S. J. Am. Chem. Soc. 1986, 108, 293. (c) Roush, W. R.; Ando, K.; Powers, D. B.; Palkowitz, A. D.; Halterman, R. L. J. Am. Chem. Soc. 1990, 112, 6339. (d) Rauniyar, V.; Zhai, H.; Hall, D. G. J. Am. Chem. Soc. 2008, 130, 8481. (e) Jain, P.; Antilla, J. C. J. Am. Chem. Soc. 2010, 132, 11884.

(3) (a) Jadhav, P. K.; Woerner, F. J. Tetrahedron Lett. 1994, 35, 8973. (b) Burgess, K.; Chaplin, D. A.; Henderson, I.; Pan, Y. T.; Elbein, A. D. J. Org. Chem. 2002, 57, 1103. (c) Ramachandran, V. P.; Subash Chandra, A. J.; Reddy, M. V. R. J. Org. Chem. 2002, 67, 7547. (d) Yin, N.; Wang, G.; Qian, M.; Negishi, E. Angew. Chem. Int. Ed. 2006, 45, 2916. (e) Penner, M.; Rauniyar, V.; Kaspar, L. T.; Hall, D. G. J. Am. Chem. Soc. 2009, 131, 14216.

(4) (a) Hoffmann, R. W.; Kemper, B. Tetrahedron Lett. 1981, 22, 5263. (b) Wuts, P. G. M.; Bigelow, S. S. J. Org. Chem. 1982, 47, 2498. (c) Brown, H. C.; Jadhav, P. K.; Bhat, K. S. J. Am. Chem. Soc. 1988, 110, 1535. (d) Ganesh, P.; Nicholas, K. M. J. Org. Chem.
1997, 62, 1737. (e) Hoffmann, R. W.; Krüger, J.; Brückner, D. New J. Chem. 2001, 102. (f) Muñoz-Hernández, L.; Soderquist, J. A. Org. Lett. 2009, 11, 2571.

(5) Yamamoto, E.; Takenouchi, Y.; Ozaki, T.; Ito, H. J. Am. Chem. Soc. 2014, 136, 16515.

(6) Representative examples of the routes used to synthesize the acetal and acylal substrates

\section{(a) A Synthesis of Z-Allyl Dibenzyl Acetal (Scheme 6)}

The allyl acetal substrates were synthesized over several steps (a). The synthesis started from commercially available propargyl diethyl acetal, which was subjected to an acid-catalyzed acetal-exchange reaction with benzyl alcohol to give the corresponding dibenzyl acetal. The subsequent deprotonation of the alkyne moiety, followed by the alkylation of the alkynyl lithium and partial reduction of the carbon-carbon triple bond gave the allyl acetal substrate. Although the exchange reaction generally proceeded in high yield, the subsequent alkylation of the terminal alkyne with an alkyl halide was typically low-yielding.

(b) A Synthesis of Z-Allyl Acylal (Scheme 7)

In contrast to the acetal substrates, the acylal substrates were much easier to prepare (b). The formylation of a terminal alkyne, followed by the gem-diacetylation of the resulting carbonyl moiety provided the corresponding propargyl acylals in moderate to high yields. The subsequent $Z$-selective reduction of the alkyne moiety in these propargyl acylals yielded the desired allylic substrates.

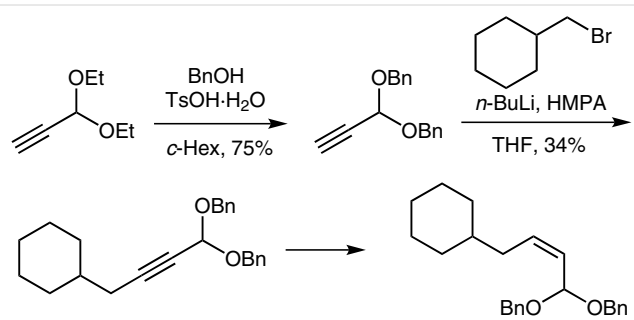

Scheme 6

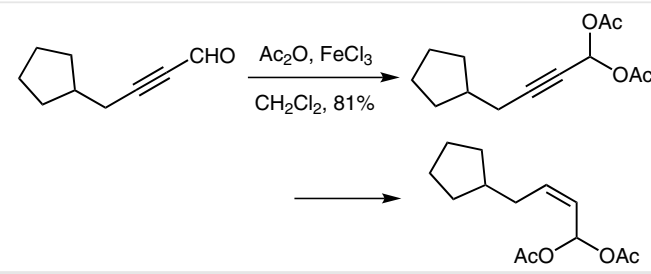

Scheme 7

(7) (a) van Heerden, F. R.; Huyser, J. J.; Williams, D.; Holzapfel, C. W. Tetrahedron Lett. 1998, 39, 5281. (b) Trost, B. M.; Lee, C. B. J. Am. Chem. Soc. 2001, 123, 3687. (c) Trost, B. M.; Lee, C. B. J. Am. Chem. Soc. 2001, 123, 3671.

(8) (a) Sydnes, L. K.; Sandberg, M. Tetrahedron 1997, 53, 12679. (b) Sandberg, M.; Sydnes, L. K. Tetrahedron Lett. 1998, 39, 6361. (c) Sandberg, M.; Sydnes, L. K. Org. Lett 2000, 2, 687.

(9) Wuts, P. G. M.; Greene, T. W. Protective Groups in Organic Synthesis; Wiley Interscience: Hoboken, 2007, 4th ed..

(10) Kavala, V.; Patel, B. K. Eur. J. Org. Chem. 2005, 441.

(11) Tamura, K.; Sugiya, M.; Yoshida, K.; Yanagisawa, A.; Imamoto, T. Org. Lett. 2010, 12, 4400. 
(12) (a) Ito, H.; Kawakami, C.; Sawamura, M. J. Am. Chem. Soc. 2005, 127, 16034. (b) Ito, H.; Ito, S.; Sasaki, Y.; Matsuura, K.; Sawamura, M. J. Am. Chem. Soc. 2007, 129, 14856.

(13) Typical Procedure for the Enantioselective Boryl Substitution of Allyl Acylals

$\mathrm{CuCl}$ (2.6 mg, $0.026 \mathrm{mmol}),(R, R)-\mathrm{BenzP}^{*}(7.2 \mathrm{mg}, 0.026 \mathrm{~mol})$, $\mathrm{B}_{2}(\text { pin })_{2}(254.8 \mathrm{mg}, 1.00 \mathrm{mmol})$, and $\mathrm{KOt}-\mathrm{Bu}(84.3 \mathrm{mg}, 0.75$ $\mathrm{mmol}$ ) were placed in a screw-capped test tube in a glove box under an argon atmosphere. After the vial was sealed with a screw cap containing a Teflon-coated rubber septum, the test tube was removed from the glove box and connected to a vacuum/nitrogen manifold through a needle. Then, dry DMI (1.0 $\mathrm{mL}$ ) was added to the mixture via a syringe with stirring at r.t. After 15-30 min, acylal (Z)-1a (129.5 mg, $0.5 \mathrm{mmol})$ was added to the reaction mixture with vigorous stirring at $0{ }^{\circ} \mathrm{C}$. After the completion of the reaction, the mixture was directly filtered through a short silica gel column with hexane-EtOAc (90:10) as the eluent. After removal of the solvents under reduced pressure, NMR yield was determined by ${ }^{1} \mathrm{H}$ NMR analysis of the crude reaction mixture $[(S, E)-\mathbf{2 a} ; \mathbf{7 9} \%]$ by using mesitylene (26.7 $\mathrm{mg}, 0.22 \mathrm{mmol}$ ) as the internal standard. The crude product was purified with flash chromatography $\left(\mathrm{SiO}_{2}\right.$, hexane$\mathrm{Et}_{2} \mathrm{O}=100: 0$ to $90: 10$ ) to give the corresponding $\gamma$-acetoxyallylboronate $(S, E)-2 a(84.8 \mathrm{mg}, 0.257 \mathrm{mmol}, 52 \%$ isolated yield).

${ }^{1} \mathrm{H}$ NMR $\left(392 \mathrm{MHz}, \mathrm{CDCl}_{3}\right): \delta=1.25(\mathrm{~s}, 12 \mathrm{H}), 1.63-1.93(\mathrm{~m}, 3 \mathrm{H})$, $2.11(\mathrm{~s}, 3 \mathrm{H}), 2.52-2.71(\mathrm{~m}, 2 \mathrm{H}), 5.45(\mathrm{dd}, J=9.4,12.5 \mathrm{~Hz}, 1 \mathrm{H})$, 7.09 (d, $J=12.2 \mathrm{~Hz}, 1 \mathrm{H}), 7.13-7.31(\mathrm{~m}, 5 \mathrm{H}) .{ }^{13} \mathrm{C} \mathrm{NMR}(99 \mathrm{MHz}$, $\left.\mathrm{CDCl}_{3}\right): \delta=20.7\left(\mathrm{CH}_{3}\right), 22.9(\mathrm{br}, \mathrm{BCH}), 24.6\left(\mathrm{CH}_{3}\right), 24.7\left(\mathrm{CH}_{3}\right)$, $32.8\left(\mathrm{CH}_{2}\right), 35.0\left(\mathrm{CH}_{2}\right), 83.4(\mathrm{C}), 115.4(\mathrm{CH}), 125.6(\mathrm{CH}), 128.2$ (CH), $128.4(\mathrm{CH}), 135.2(\mathrm{CH}), 142.3(\mathrm{C}), 168.1(\mathrm{C})$. HRMS (EI): $\mathrm{m} / z[\mathrm{M}]^{+}$calcd for $\mathrm{C}_{19} \mathrm{H}_{27} \mathrm{BO}_{4}$ : 329.20387; found: 329.20481 . $[\alpha]_{D}^{22} .^{2}+5.4\left(c 1.0, \mathrm{CHCl}_{3}, 95 \%\right.$ ee $)$. The ee value was determined by HPLC analysis [Daicel CHIRALPAK OD-3, 2-PrOH-hexane = $\left.0.25: 99.75,0.5 \mathrm{~mL} / \mathrm{min}, 40{ }^{\circ} \mathrm{C}\right]: t_{\mathrm{R}}$ (major) $=25.44 \mathrm{~min} ; t_{\mathrm{R}}$ $($ minor $)=24.83 \mathrm{~min}$.

(14) Kobayashi, S.; Endo, T.; Schneider, U.; Ueno, M. Chem. Commun. 2010, 46, 1260.

(15) (a) Carosi, L.; Lachance, H.; Hall, D. G. Tetrahedron Lett. 2005, 46, 8981. (b) Rauniyar, V.; Hall, D. G. J. Am. Chem. Soc. 2004, 126, 4518. (c) Ishiyama, T.; Ahiko, T.-A.; Miyaura, N. J. Am. Chem. Soc. 2002, 124, 12414. (d) Kennedy, J.; Hall, D. G. J. Am. Chem. Soc. 2002, 124, 11586. 